\title{
Light Dark Matter search in a beam-dump experiment: BDX at Jefferson Lab
}

\author{
Mariangela Bondí ${ }^{1, a}$ for the BDX Collaboration \\ ${ }^{1}$ INFN - Catania, Via S. Sofia 64, 95125 Catania (Italy)
}

\begin{abstract}
The Beam Dump eXperiment (BDX) aims to investigate the existence of light Dark Matter (DM) particles in the $\mathrm{MeV}-\mathrm{GeV}$ mass range, theoretically well motivated but still barely explored. In this mass range BDX will be sensitive to large regions of DM parameter space, exceeding the discovery potential of existing and planned experiments by up to two orders of magnitude.

BDX is an $\mathrm{e}^{-}$-beam thick-target experiment, expected to run at Jefferson Lab, downstream of the Hall A beam dump, receiving up to $10^{22}$ electron-on-target (EOT). The detector is made of two main components: an electromagnetic calorimeter (Ecal), and a veto detector used to suppress the background. BDX will be sensitive to elastic and inelastic DM-electron scattering at the level of 10 counts per year, reaching the limit of the irreducible background due to beam-produced neutrinos. The expected signature of the DM interaction in the Ecal is $\mathrm{a} \sim \mathrm{GeV}$ electromagnetic shower paired with a null activity in the surrounding active veto counters.

In order to evaluate the background contributions two approaches were used: the cosmogenic background was extrapolated from results obtained with a prototype running at INFN-LNS (Italy), while the beam- related background was evaluated by GEANT4 Monte Carlo simulations.

This paper describes the experimental setup and the results of the background studies along with the status and prospectives of the experiment.
\end{abstract}

\section{Introduction}

The search for Dark Matter (DM) is one of the hottest topics of modern physics. Despite the copious number of astrophysical and cosmological observations proving its existence, its elementary properties remain largely elusive [1]. Although the Standard Model (SM) contains several neutral particles, none serves as a viable dark matter candidate. Thus the existence of DM is an overwhelming evidence of physics beyond the SM and uncovering its particles identity is top priority in fundamental physics.

Various extensions of the SM propose different candidates for the role of DM particles, like the Weakly Interacting Massive Particles (WIMPs) $(\sim 10 \mathrm{GeV}-10 \mathrm{TeV}$ mass range) expected to weakly interact with SM particles [2]. Due to the lack of evidence for WIMPs so for from LHC and direct DM searches, other well motivated models of DM gained recently the interest of the physics community. These models take into account DM particles in MeV-GeV mass range (Light Dark Matter (LDM)), whose existence is motivated by both empirical and theoretical considerations. On the theory side, simple SM extensions can accommodate DM-SM interactions that yield the observed DM cosmological abundance. On the empirical side, such models feature particles that explain the currently discrepant value of the muon's anomalous magnetic moment and resolve anomalies in astrophysical observations, while simultaneously evading cosmological

\footnotetext{
a e-mail: mariangela.bondi@ct.infn.it
}

and direct-production constraints.

By and large the most viable LDM model to probe is that where LDM interacts with the SM through a "dark photon" $A^{\prime}$. Dark photon can mix with the ordinary photon through kinetic mixing, which induces their weak coupling to electric charge, $\epsilon e$, where $\epsilon<10^{-3}$ [3][4]. The minimal parameter space of vector-mediated LDM is characterized by $\epsilon$, the coupling $\alpha_{D}$ of the $A^{\prime}$ to the LDM particle $\chi$ and two masses $m_{A^{\prime}}$ and $m_{\chi}$.

In this scenario, LDM searches at collider are limited by the huge SM backgrounds producing missing energy.

Instead, high intensity $\sim \mathrm{GeV}$ electron-beam fixed-target experiments offer large sensitivity to a broad class of dark sector scenarios that feature particles in the elusive MeV$\mathrm{GeV}$ mass range [5]. Electron beam-dump experiments are complementary to dedicated efforts at proton beam facilities, have comparable DM scattering yield, can run on a smaller scale than proton-beam counterparts, and benefit of reduced beam-related backgrounds.

A generic sketch of a beam dump experiment is shown in Fig.1. The interaction between the energetic electrons and the atoms of the dump leads to the production of Dark photons through a Bremsstrahlung-like radiative process. Subsequently, the $A^{\prime}$ decays to LDM particle-antiparticle pairs $(\chi \bar{\chi})$ (see Fig.2a), which unaltered pass through the dump and the shielding between the dump and the detector. A fraction of them will then scatter off nucleons, nuclei, or electrons in the detector volume, giving rise to a detectable signal. For $\mathrm{e}^{-}-\chi$ interaction (see Fig.2b), since 
$m_{e}<m_{\chi}$, the typically scattered electron carries GeVscale energy producing an electromagnetic shower in the detector. For $\chi-\mathrm{N}$ interactions the much higher mass of nucleons result in a typically recoil energy of the order of $\sim \mathrm{MeV}$.

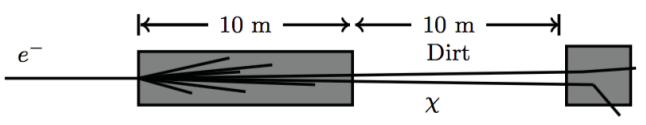

Beam

Dump Detector

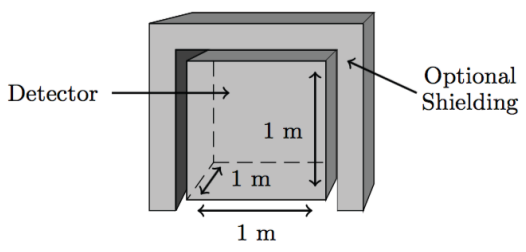

Figure 1. Schematic of the experimental setup. A high-intensity multi-GeV electron beam impinging on a beam-dump produces a secondary beam of dark sector states. In the basic setup, a small detector is placed downstream the beam-dump so that muons and energetic neutrons are entirely ranged out.

The considerable sensitivity of beam-dump experiments to LDM is underscored by the reach of running neutrino experiments [6-9] . For example, LSND measurement of meson production and decay [10] can be used to derive the most stringent constraints to date on the parameter space for invisibly-decaying dark mediators that couple to both baryons and leptons [9].

a)

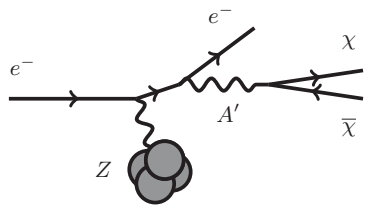

b)

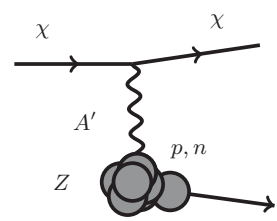

Figure 2. a) $\chi \bar{\chi}$ pair production in electron-nucleus collisions via the Cabibbo-Parisi radiative process (with $A^{\prime}$ on - off-shell) and b) $\chi$ scattering off an electron in the detectors

The Beam Dump experiment (BDX) at JeffersonLab [11] aims to produce and detect LDM, assuming valid the above cited theoretical paradigm. Tacking advantage of the high intensity $\sim \mathrm{GeV}$ electron beam available at JLab, $\mathrm{BDX}$ has the unique capability of dramatically improve sensitivity to $\mathrm{MeV}$ - GeV DM, extending well beyond the reach of existing and proposed experiments, reaching the limit of the un-reducible background due to beamproduced neutrinos.

\section{BDX at Jefferson Lab}

\subsection{Experimental setup}

BDX at Jefferson Lab will make use of the CEBAF (Continuous Electron Beam Accelerator Facility) highintensity electron beam, impinging on the JLab Hall-A beam dump which is enclosed in a concrete tunnel at the end of the beam transport line. The Hall-A at JLab can receive from CEBAF a $11 \mathrm{GeV}$ electron beam with a maximum current of about $65 \mu \mathrm{A}$. The possibility of running to the maximum energy available at Jlab together with a sizeable current, that allows to collect $10^{22}$ electron-on-target (EOT) in 285 days, in full parasitic runs, makes Hall-A the optimal choice.

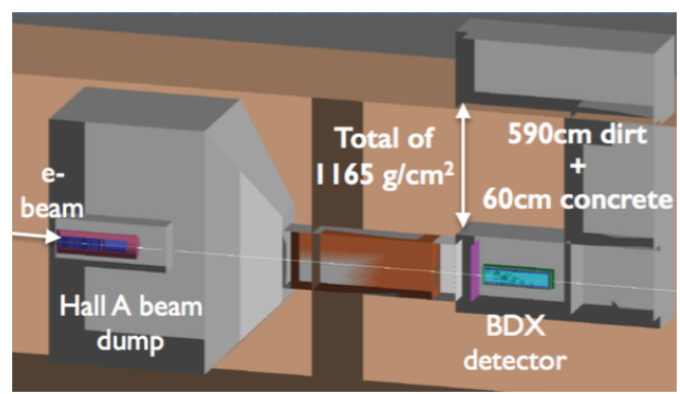

Figure 3. The BDX experimental setup.

The BDX detector will be placed along the LDM beam trajectory, in a new underground facility located 20 meters downstream of the Hall-A dump (Fig. 3). The extremely low signal rate expected, due to the two-step processes involving weak mixing between the SM photon and $A^{\prime}$, makes background rejection the critical issue of BDX. To range out all the SM beam products, except neutrinos, the space between the dump and the detector will be filled with dirt plus $\sim 7 \mathrm{~m}$ of concrete and $\sim 7 \mathrm{~m}$ of iron; to reduce the beam-unrelated background, mainly due to cosmic neutrons and muons, the detector will be placed in a bunker 8 $\mathrm{m}$ underground with 10 meters of water equivalent (mwe) overburden. Moreover, the detector will include an active veto and passive shielding.

The detection of the low-energy scattered protons is a secondary goal of the experiment because it provides an alternative probe of LDM production. However, background at $\sim \mathrm{MeV}$ energy scale expected to limit the sensitivity in this channel.

\subsection{Detector design}

The BDX detector is made of two main components: an electromagnetic calorimeter used to detect signals pro- 
duced by the interacting DM particles, and a veto detector used to reduce the cosmogenic background (see Fig.4 for a sketch of the detector). The calorimeter consists of 8 modules of $10 \times 10 \mathrm{CsI}(\mathrm{Tl})$ crystals each, arranged with the long size along the beam direction. The average size of each crystal is $(4.7 \times 5.4 \times 32.5) \mathrm{cm}^{3}$. This arrangement has a cross section of $50 \times 55 \mathrm{~cm}^{2}$ for a total length of 295 $\mathrm{cm}$. SiPM are used as light-readout sensors.

The BDX EM calorimeter is operated inside two hermetic layers of active veto counters, made of plastic scintillators: the outermost called Outer Veto (OV) and the innermost Inner Veto (IV). The OV consists of $2 \mathrm{~cm}$-thick plastic scintillators coupled to PMT through plexiglass light guides or wavelength shifting plastic. Due to the large size requested to cover the whole calorimeter and to preserve the possibility of changing the geometry, the OV is segmented in many different paddles. In particular, the top and the bottom layers are divided in two parts while the lateral sides are made by 11 paddles per side.
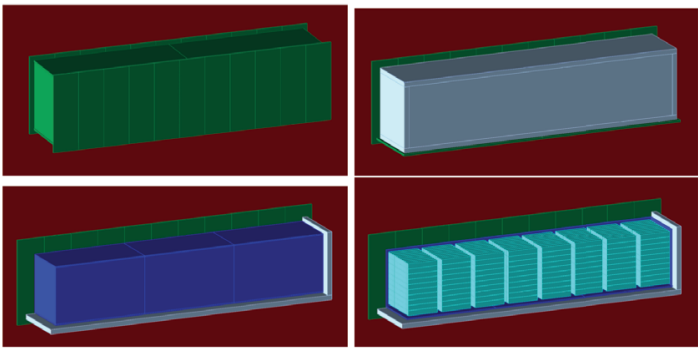

Figure 4. The BDX detector. The Outer Veto is shown in green, the Inner Veto is blue and the lead vault in gray. Crystals arranged in 8 blocks of 10x10, are shown in light blue. Each crystal is read by a SiPM directly attached to the front face (not shown).

The IV consists of a hermetic box comprised of six 1-cm thick plastic scintillator paddles. The lateral paddles are read on one side by $4 \mathrm{SiPMs}$, directly glued to the scintillator surface. Grooves on the surface host two $1 \mathrm{~mm}$ WLS fibers to convey the scintillation light to the SiPM. The upstream and downstream covers have a spiral-like groove. The WLS fibers are read by one SiPM per paddle located on the scintillator surface.

A $5 \mathrm{~cm}$ thick layer of lead is placed between the two active vetoes. The "sandwich" configuration, with the lead between the IV and the OV, has been chosen to detect with the OV the cosmic muons that may produce spallation in the lead. It also acts as a radiator for high energy photons, allowing the detection of charged secondaries in the IV.

This detector concept has been optimized and validated in various campaign of measurements at INFN - Sezione di Catania and Laboratori Nazionali del Sud (LNS) with two prototypes described in Section 3 and 4.

\subsection{Expected Background}

\subsubsection{Beam-Related Background}

The beam-related background for the electron recoil measurements was studied through MC simulations, considering a detection threshold of $300 \mathrm{MeV}$. The interaction of the primary $11 \mathrm{GeV}$ electron beam in the dump was simulated with a GEANT4-based code and the flux of secondaries was studied as a function of the distance from the dump. For this purpose, the flux of particles was sampled over planes perpendicular to the beam direction. Results are shown in Fig. 5: $\mathrm{Z}=0$ corresponds to the upstream end of the aluminum dump, while the detector is located at $\mathrm{Z}=20 \mathrm{~m}$. The only particles that are found to reach the detector area are $v$ and $\bar{v}$, predominantly from pion and muon decays at rest. In fact, the number of neutrons and muons decreases rapidly as the distance from the dump increases and, performing an exponential extrapolation of the projected counts, we estimated that no neutrons and muons would reach the detector with energy $\geq 300 \mathrm{MeV}$.

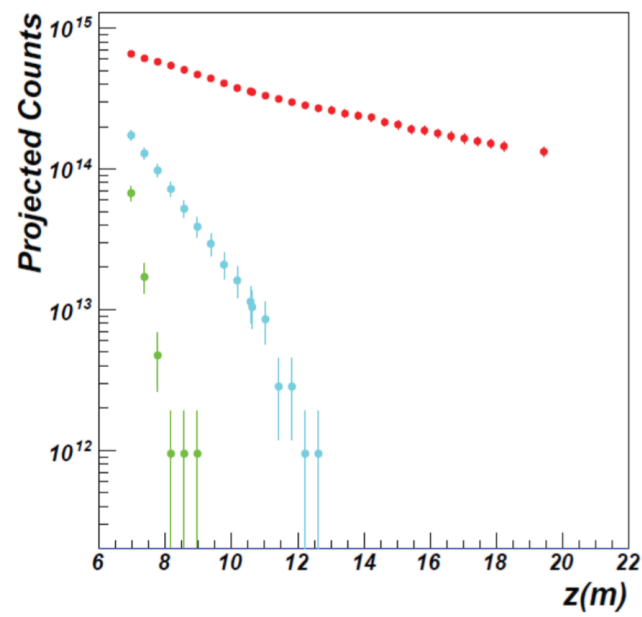

Figure 5. Number of secondary particles produced by the interaction of the $11 \mathrm{GeV}$ electrons as a function of the distance from the upstream end of the dump. The reported counts are normalized to $10^{2} 2$ EOT. The threshold on the particles energy is 500 $\mathrm{MeV}$. The different colors correspond to different particle types: neutrinos in red, muons in cyan, neutrons in green and photons in blue.

The neutrino reaching the detector are mostly $v_{\mu}$ and $\bar{v}_{\mu}$, with only about $3-4 \% v_{e}$ and $\bar{v}_{e}$. In this energy range, neutrinos interact mostly via charged current, with the dominant processes being quasi-elastic scattering and resonance production on the nucleon, whose cross section is of the order of $\sigma_{v N} \sim 10^{-38} \mathrm{~cm}^{2}$ [12]. These neutrinos can scatter in the BDX detector producing a muon or electron that can be detected. Muons with energy of order of hundreds of MeV loose energy via ionization and can be distinguished from the electron recoil signal. The scattered 
electrons instead, induce electromagnetic showers, resulting in the same signature of the $\chi-\mathrm{e}^{-}$interaction. The result of the simulation indicates that, for an energy threshold of $300 \mathrm{MeV}$, the expected neutrino background is of order of $\sim 10$ events for $10^{22}$ EOT.

\subsubsection{Cosmogenic Background}

Beam-unrelated background is mainly due to cosmic neutrons, cosmic muons and their decay products, including rare decays of muons producing gamma's between the passive shielding and the active veto. Both direct cosmic flow and secondary particles contribute to the beam-unrelated background rate in the detector.

This rate has been evaluated by extrapolating the results obtained with the BDX prototype in Catania. The similar experimental set-up (including overburden) as well as a full prototype that incorporates all the elements of the BDX detector (active veto's, lead shielding, CsI(Tl) crystal) provide a solid base for a realistic, although conservative, estimate of the expected rates. Details of the experimental conditions and data analysis are reported in the next sections. The extrapolation has been performed by scaling the experimental rates of a single crystal to the 800 crystals comprising the full detector. This is certainly an upper limit on the expected rates since this assumes crystal-to-crystal fully uncorrelated counts, which overestimates the case for $\chi-\mathrm{e}^{-}$scattering. The results show that for energy thresholds higher enough, between 300$350 \mathrm{MeV}$, the number of cosmogenic background counts, in 285 days, reduces to zero. Thus, by choosing the appropriate energy threshold we could expect to have zero background. However, in order to be conservative, we considered a value of cosmic events $N_{\text {cosmic }}=3$ in the evaluation of the BDX reach and sensitivity.

\subsection{BDX Expected Reach}

In this Section we present an example of the expected reach of BDX for the $\chi-\mathrm{e}^{-}$channel. Results are reported as upper limit on exclusion plot. More excluding plots for different parameter spaces and/or DM scenarios, together with the detail of the analysis, are reported in [11]. The regions above the lines are excluded since the model, with the chosen parameters, predicts a number of counts greater than two times the sigma of the background. This is true in case of null results. If any excess is observed, a thorough statistical analysis will be necessary to claim a positive result.

In case of no positive observation, the accumulated data would provide very stringent limits on the DM parameter space. In Fig. 6 we show the BDX reach in the $\epsilon^{2}-m_{A^{\prime}}$ parameter space (for $m_{\chi}<<m_{A^{\prime}}$ ). The gray region is excluded by the re-analysis of old data from non-optimized experiments. The red curves show 3, 10 and 20 event BDX yield projections, including the detection efficiency estimated with MC simulations, for electron scattering with $10^{22}$ EOT and a $300 \mathrm{MeV}$ recoil energy threshold.

As shown in Fig. 6, BDX at JLAB extends considerably the possible reach with respect to previous experiments, probing wide unexplored DM parameter space regions.

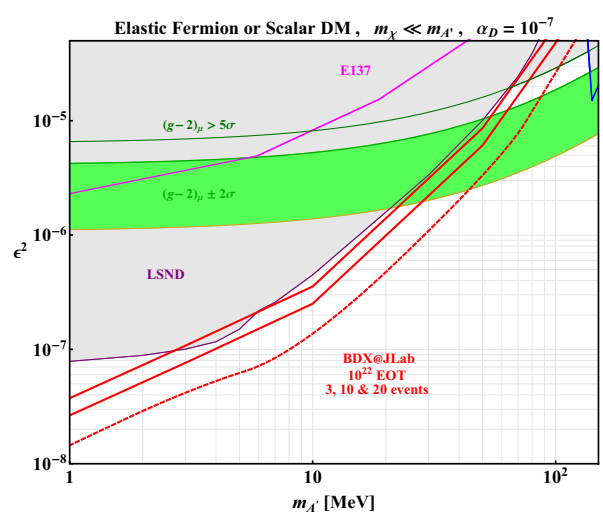

Figure 6. Red curves show 3, 10, 20 event BDX yield projections for $\chi \mathrm{e}^{-}$scattering for $10^{22}$ EOT and a $300 \mathrm{MeV}$ recoil energy threshold. Grey regions have already been ruled out by previous experiments.

\section{Cosmic measurements campaign in Catania}

In this section, we report some details and the results of the dedicated campaign of measurements performed in Catania, Italy (INFN - CT) and Laboratori Nazionali del Sud (INFN - LNS) with first prototype of the BDX detector. These measurements provided information useful for the full detector design and its expected performance in a realistic configuration. We tested the proposed technologies: CsI(Tl) crystals read by SIPMs, plastic scintillators read by PMTs for the OV and plastic scintillator coupled to SIPMs by WLS fibers for the IV; we checked the effect of the lead shielding and the overburden, we measured the single crystal rates as a function of the energy threshold in combination (anti-coincidence) with the veto systems. In order to test and commission whole setup the data were initially taken exposing the prototype to cosmic rays with minimal shielding (15 cm of concrete roof at INFN-CT). Then the prototype was moved into a bunker at LNS with a concrete overburden of $470 \mathrm{~cm}$ corresponding to an effective thickness of $1080 \mathrm{~g} / \mathrm{cm}^{2}$ similar to $1165 \mathrm{~g} / \mathrm{cm}^{2}$ expected at JLab.

\subsection{The BDX prototype}

The first BDX prototype was made by a single $\mathrm{CsI}(\mathrm{Tl})$ crystal surrounded on all sides by a layer of veto detectors (IV), a vault of lead bricks, and a second layer of veto detectors forming the OV. The combined use of two charged-particle veto-counter systems allows to compensate for their inefficiencies and better reject background events. Between the inner and the outer veto, the $5 \mathrm{~cm}$ thick lead vault shields the crystal from radiogenic low energy gammas. 


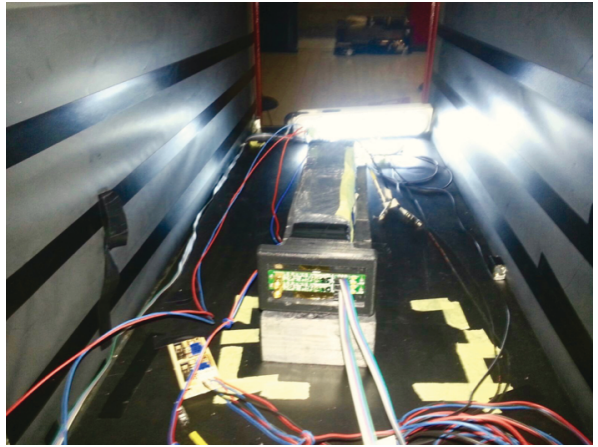

Figure 7. The CsI(Tl) crystal inside the first prototype. The two charge-preamplifiers coupled to the SiPMs are visible.

The CsI(Tl) crystal, shown in Fig. 7, is $31 \mathrm{~cm}$ long and has a trapezoidal shape with a $4.7 \times 4.7 \mathrm{~cm}^{2}$ front-face and a $6 \times 6 \mathrm{~cm}^{2}$ back-face. Two $3 \times 3 \mathrm{~mm}^{2}$ SiPMs (Hamamatsu S13360-3025CS and S13360-3050CS), with pixel size of 25 and $50 \mu \mathrm{m}$, are glued to the crystal front-face and connected each to a custom trans-impedance amplifier.
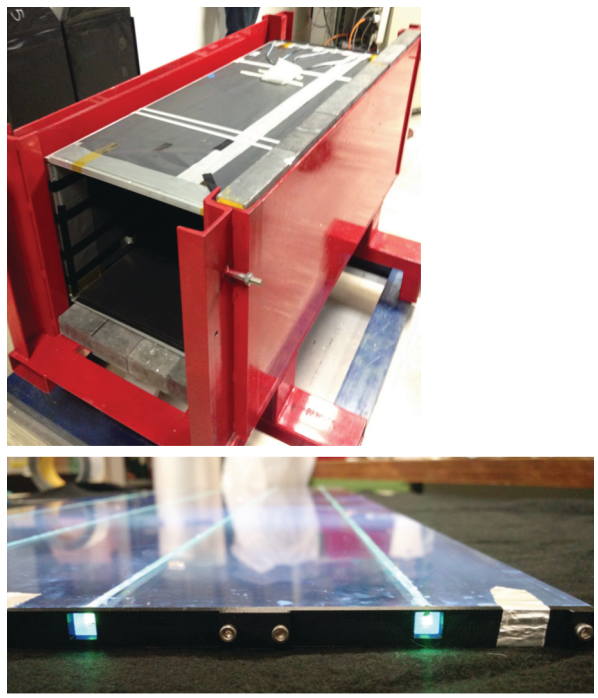

Figure 8. A picture of the Inner Veto mounted inside the mechanical structure of the prototype (top) and a picture of the top paddle (bottom).

The IV, shown in Fig. 8 is made by plastic scintillators, 1 $\mathrm{cm}$ thick, forming a nearly hermetic parallelepiped. Two $35 \times 42 \mathrm{~cm}^{2}$ EJ200 scintillators are used for the downstream and upstream caps. On each of them a spiral groove hosts a WLS fiber used to collect and transfer the light to a SiPM. Three $35 \times 140 \mathrm{~cm}^{2}$ EJ200 scintillators form the top, left and right sides of the veto. In this case, the WLS fibers are inserted into four linear grooves running parallel to the long side of the plastic. This solution results in an high detection efficiency ( $>99.5 \%$ ), almost independent on the hit point. Finally, in order to test another possible technology for the IV, the bottom side was made by four bars of extruded plastic scintillators, $8 \times 140 \mathrm{~cm}^{2}$ each, individually readout by a SiPM coupled to WLS fibers inserted in the middle of each bar. The Outer Veto, shown in Fig. 9 , is made by $2 \mathrm{~cm}$ thick NE110 plastic scintillators. The top (bottom) side is made by two $80 \times 40 \mathrm{~cm}^{2}$ paddles. Three scintillators of the same area are vertically arranged to cover each of the two lateral sides. A "fish tail" shaped PMMA light guide, glued on one side of the scintillator, directs the light to a 2" photomultiplier tube (Thorn EMI 9954A) optically matched with the light guide trough optical grease. A smaller paddle $\left(56 \times 50 \mathrm{~cm}^{2}\right)$ forms the upstream (downstream) cap. In this case, light is readout by a 1" Photomultiplier tube (R1924A Hamamatsu) placed in the middle of the plastic surface and directly coupled to it through optical grease. A detection efficiency $>99.5 \%$, uniform over the whole scintillator surface, was measured for each $\mathrm{OV}$ paddle for cosmic rays selected by triggering on the coincidence of two small paddles placed above and below the scintillator.

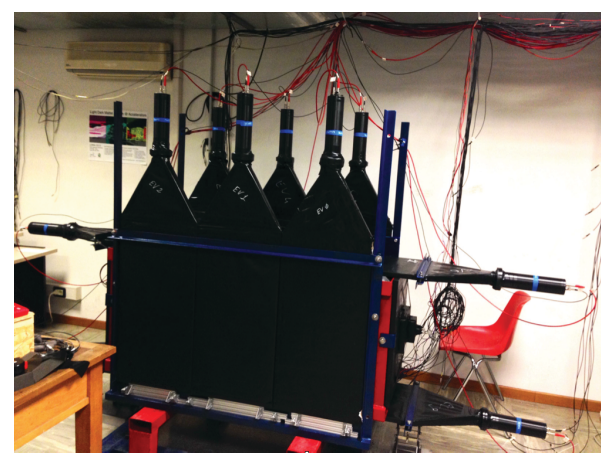

Figure 9. The prototype mounted and cabled at the INFNSezione di Catania. OV detectors are visible

Data acquisition is based on VME-VXS JLab fa250 digitizers with 12 bit resolution, $250 \mathrm{MHz}$ sampling rate and $2 \mu$ s readout window. The main trigger is generated by a signal over threshold in the CsI(Tl) crystal, namely from the logic OR of the two SIPMs. The output signals are split by a $50 \mathrm{Ohm}-50 \mathrm{Ohm}$ divider: one copy is sent to the FADC and the other one to a Leading-Edge Discriminator with thresholds set to $20 \mathrm{mV}$ and $50 \mathrm{mV}$ for the 25 and 50 $\mu \mathrm{m}$ SiPMs, respectively. These thresholds correspond to about 5 photo electrons (phe) for both SIPMs and to an energy threshold for protons of about $2 \mathrm{MeV}$.

\subsection{Cosmic measurement campaign at LNS - INFN}

The primary goal of the LNS campaign was to measure the absolute rates of cosmogenic background in a experimental condition similar to that expected at JLAB. The measurement campaign started in April 2016: the results presented here correspond to about 1 month of data-taking. 
The following data-reconstruction procedure was adopted. For the CsI crystal, both waveforms of the SiPM were numerically integrated over a gate of $2 \mu$ s to obtain the corresponding charge, that was converted in $\mathrm{MeV}$ units by using calibration constants deduced from the cosmic-rays Landau distribution most probable value. For the IV paddles with multiple readout we required, after numerical integration of the waveforms, that at least two SiPM of the same counter collect 2.5 phe each, within a coincidence gate of $100 \mathrm{~ns}$. For the IV counter with a single SiPM, an higher threshold ( $\sim 12.5$ phe) was implemented. For the OV each PMT signal was numerically integrated, and after this a $100 \mathrm{keV}$ energy threshold was applied.

We studied events with the following topologies:

- All events;

- Anti-coincidence with IV;

- Anti-coincidence with OV;

- Anti-coincidence with both IV and OV;

where anti-coincidence means that we selected events with no activity in any of the veto counters.

Results corresponding to the low-energy part of the CsI energy spectrum are shown in Fig. 10.

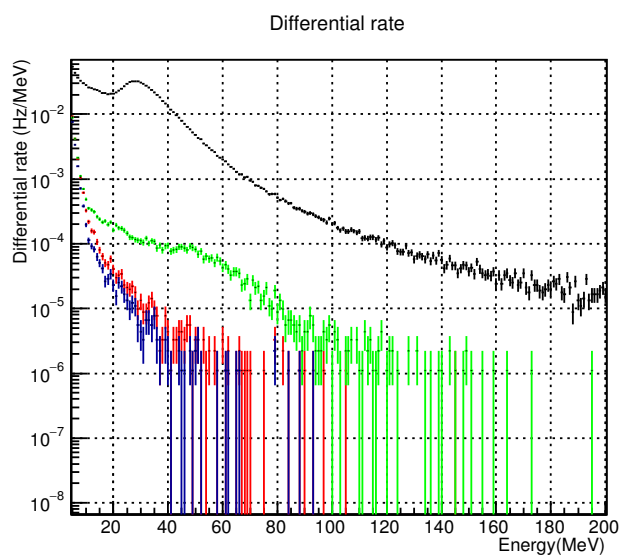

Figure 10. Measured event rate as a function of deposited energy in the crystal. Different colors refer to the different anti-coincidence selections: black -all events, red -anticoincidence with IV, green - anti-coincidence with OV, blue anti-coincidence both veto systems.

The integrated rate, requiring the anti-coincidence with both veto systems, for a $10 \mathrm{MeV}(20 \mathrm{MeV})$ threshold, is $1.7 \mathrm{mHz}(0.37 \mathrm{mHz})$.

The analysis of the high-energy part of the spectrum $(\mathrm{E}>100 \mathrm{MeV})$ is, instead, more critical, due to the low statistics. To obtain a conservative estimate of the high energy background, we proceeded performing an extrapolation from the lower part of the spectrum (where a nonzero event rate was measured) to the higher regime, trough a Maximum Likelihood fit, using an exponential function. The systematic error has been evaluated by changing the fit range and comparing the obtained results (see
Fig. 11 for the differential rate extrapolated for the IV anticoincidence events).

Results are reported in Tab.1 for the inner-veto anticoincidence selection and for different energy thresholds.

Table 1. The inner-veto anti-coincidence rate for the BDX-prototype, measured in the LNS configuration, obtained by extrapolating from the low energy part of the spectrum.

\begin{tabular}{|c|c|}
\hline Energy threshold & Extrapolated rate \\
\hline $200 \mathrm{MeV}$ & $(3.6 \pm 1.5) \cdot 10^{-8} \mathrm{~Hz}$ \\
\hline $250 \mathrm{MeV}$ & $(2.9 \pm 1.3) \cdot 10^{-9} \mathrm{~Hz}$ \\
\hline $300 \mathrm{MeV}$ & $(2.4 \pm 1.1) \cdot 10^{-10} \mathrm{~Hz}$ \\
\hline $350 \mathrm{MeV}$ & $(1.9 \pm 0.9) \cdot 10^{-12} \mathrm{~Hz}$ \\
\hline
\end{tabular}

These results were used to extrapolate the expected background rates of the final BDX experiment, scaling the experimental rates of a single crystal to the 800 crystals comprising the full detector. As already pointed out in section 2.3.2, this is an upper limit on the expected rates since this assumes crystal- to-crystal fully uncorrelated counts, which overestimates the case for $\chi-\mathrm{e}^{-}$scattering. However, these rates allowed to obtain a conservative evaluation the final BDX reach reported in Fig.6.

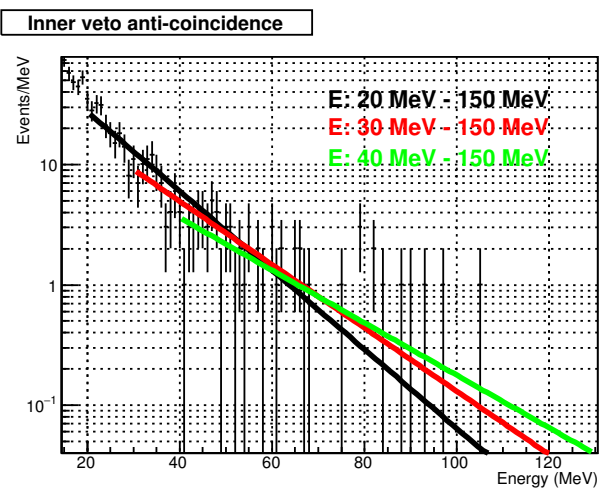

Figure 11. Extrapolation of the measured CsI energy spectrum in anti-coincidence with the IV from low-energy to high-energy region, where no events were detected. The three curves refer to Maximum Likelihood fits performed in different energy ranges.

\section{Upgrade of the prototype and the second cosmic measurements campaign in Catania}

BDX was proposed to JLab Program Advisory Committee (PAC) on June 2016 [11]. The PAC found the physics case exiting and conditionally approved the proposal, encouraging the collaboration to perform further studies to benchmark beam-related background simulations. In this respect, the collaboration is considering the possibility to propose on site measurements with a much smaller and 
simpler detector of the beam-produced muon flux at different distances from the dump. At the same time, the prototype detector was improved in all its components and a new cosmic measurement campaign is currently underway in Catania.

In particular, the single crystal was replaced with a $4 \times 4$ matrix of $\mathrm{CsI}(\mathrm{Tl})$ crystals (Fig. 12) assembled and tested at the INFN - Sezione di Genova, each read-out by a $6 \times 6$ $\mathrm{mm}^{2}$ SiPM (Hamamatsu S13360-6025PE).

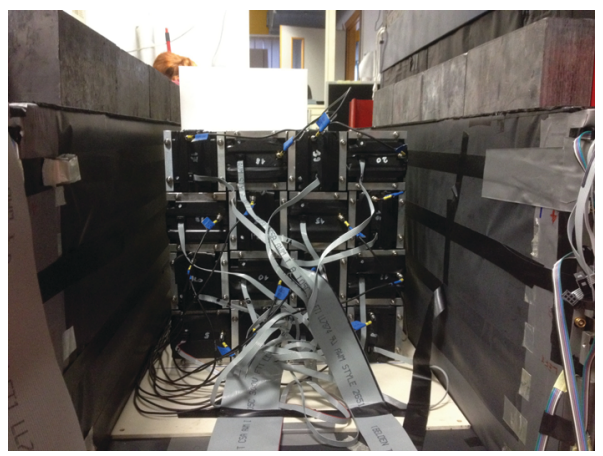

Figure 12. A picture of the $4 \times 4$ matrix of $\mathrm{CsI}(\mathrm{Tl})$ crystals placed inside the second prototype.

Important changes have also been made to the two veto systems in order to test new solutions and increase their efficiency. In the IV the 4 extruded bars of the bottom side were replaced with a more efficient clear-plastic scintillator $35 \times 140 \mathrm{~cm}^{2}$, read-out by a system of WLS and SiPM. In particular the WLS fibers are hosted into 4 linear grooves running parallel to the long side of the scintillator, and are used to collect and transfer the light to 4 independent SiPMs.

The upgraded version of the $\mathrm{OV}$ is much more hermetic thanks to the following changes: the two $80 \times 40 \mathrm{~cm}^{2}$ paddles on the top side were replaced by a single large area (184 x $54 \mathrm{~cm}^{2}$ ) plastic scintillator, read-out on both sides; the upstream and downstream caps were replaced by two new larger plastic scintillators featuring a more efficient light collection technique; one more $80 \times 40 \mathrm{~cm}^{2}$ paddle was added for each lateral side. For the new detectors (top, upstream and downstream) a different read-out solution is used, with the light guide replaced by slim wavelengthshifting plastic [13]. This solution has the advantage of reducing significantly the space and the mechanics needed by the light read-out system and making the veto more compact and hermetic, preserving, at the same time, a good light collection efficiency. Top detector comprises a large EJ200 scintillator $2.5 \mathrm{~cm}$ thick, which is diamond milled on all sides, except the two shortest ones that are 'frosted' and face one strip of EJ280 wavelength shifting plastic $\left(2.5 \times 2.5 \times 62 \mathrm{~cm}^{3}\right)$. At the end of each EJ280 is a $2.5 \mathrm{~cm}$ in diameter, Hamamatsu R1924A photomultiplier tube (PMT) for light collection. Fig.13 shows the OV top detector.

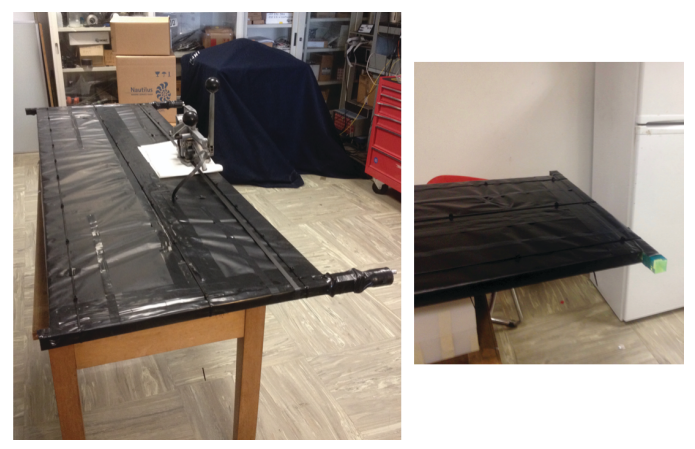

Figure 13. The OV top detector is shown. In the right panel a detail of one of the wavelength shifting plastic is shown.

In the upstream and downstream panels light is collected only on one side. The EJ200 plastic scintillators have dimensions $58 \times 66 \times 2.5 \mathrm{~cm}^{3}$, while the EJ280 wavelength-shifting plastic are $62 \times 2.5 \times 2.5 \mathrm{~cm}^{3}$, and are also coupled to Hamamatsu R1924A photomultipliers. Fig. 14 shows the upgraded BDX prototype.

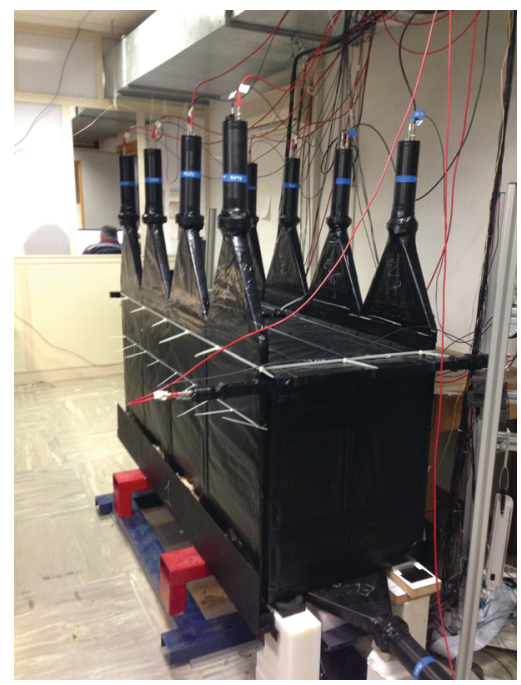

Figure 14. The prototype mounted and cabled at the INFNSezione di Catania for the second cosmic measured campain.

In Fig. 15 is shown the differential rate of the event collected by single CsI(Tl) crystal as a function of the energy for following class of events:

- all events;

- anti-coincidence of the $\mathrm{OV}$ in the old configuration (first prototype - Proto1);

- anti-coincidence of the OV in the new configuration (second prototype - Proto2);

As shown in Fig.15, thanks to the changes, the efficiency of the OV is increased by a factor $\sim 4 / 5$. 


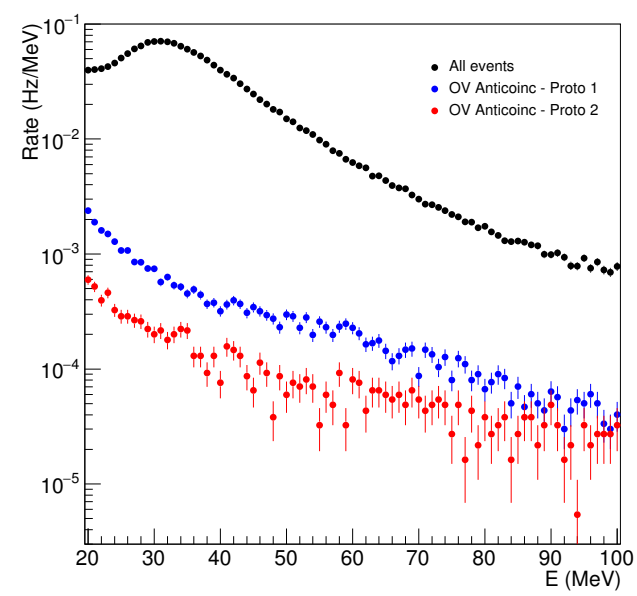

Figure 15. Measured event rate as a function of deposited energy in the crystal. Different colors refer to the anti-coincidence of the $\mathrm{OV}$ in the old configuration (blue dots) and in the new configuration (red dots).

\section{Conclusion}

BDX at Jlab aims to search for dark-matter particles in the $\mathrm{MeV}-\mathrm{GeV}$ mass range, in an electron beam dump setup. It will look for these particles using an electromagnetic calorimeter surrounded by active vetos and passive shielding in a new underground facility located downstream of the Hall-A beam dump. BDX at JLab will hit the limit of this class of experiment reaching the wall of the irreducible background produced by $\mathrm{CC}$ interactions of beam-related $v_{e}$ in the detector. The sensitivity of BDX was evaluated by measuring the cosmic background with a prototype under conditions similar to those proposed at Jlab and estimating the beam-related background using GEANT4 MonteCarlo simulations. In the absence of a signal, electromagnetic shower thresholds between 0.3 and $0.5 \mathrm{GeV}$ can be used to set limits on the production of dark matter that exceed the expected sensitivity of previous, and proposed experiments by up to two orders of magnitude.

BDX was conditionally approved by PAC44. The collaboration is considering the possibility of measuring in the near future beam-related background for benchmarking the simulations. Moreover in view of a further optimization of the experimental setup, a second prototype has been assembled and it is currently running cosmic particles in Catania. Further possible optimitations related to the front-end electronics and acquisition system are also under study.

\section{References}

[1] K. A. Olive et al., Review of Particle Physics. Chin. Phys., C38:090001, 2014.

[2] Annika H. G. Peter. Dark Matter: A Brief Review. 2012.

[3] B. Holdom, Phys. Lett. B 166B, 196 (1986).

[4] N. Arkani-Hamed et al., Phys. Rev. D 79, 015014 (2009)

[5] E. Izaguirre et al., Phys. Rev. D 90, 014052 (2014)

[6] R. Dharmapalan et al. [MiniBooNE Collaboration], arXiv:1211.2258 [hep-ex].

[7] B. Batell, M. Pospelov and A. Ritz, Phys. Rev. D 80, 095024 (2009).

[8] P. deNiverville, M. Pospelov and A. Ritz, Phys. Rev.D 84, 075020 (2011).

[9] P. deNiverville, D. McKeen and A. Ritz, Phys. Rev.D 86, 035022 (2012).

[10] L. B. Auerbach et al. [LSND Collaboration], Phys.Rev. D 63, 112001 (2001).

[11] M. Battaglieri et al., Dark matter search in a Beam-Dump eXperiment (BDX) at Jefferson Lab, arxiv:1607.01390v1.

[12] J. A. Formaggio and G. P. Zeller, Rev. Mod. Phys., 84, 1307 (2012).

[13] K.J. Thomas and et al., NIMA, 724, 47-53 (2013). 